\title{
2D and 3D Numerical Models of the Growth of Foraminiferal Shells
}

\author{
Paweł Łabaj ${ }^{1}$, Paweł Topa ${ }^{1}$, Jarosław Tyszka ${ }^{2}$, and Witold Alda ${ }^{1}$ \\ 1 Institute of Computer Sciences, AGH, al. Mickiewicza 30, 30-059 Cracow, Poland \\ \{topa, alda\}@uci.agh.edu.pl \\ 2 Institute of Geological Sciences (Cracow Research Centre), Polish Academy of \\ Sciences, ul. Senacka 1, 31-002 Cracow, Poland \\ ndtyszka@cyf-kr.edu.pl
}

\begin{abstract}
This paper focuses on modelling and visualisation of mineral shells of foraminifera (unicellular aquatic, mainly marine, organisms). Our recent model follows the moving reference approach of theoretical modelling of foraminiferal shells. This model introduces three important aspects of simulations, i.e. non-circular chamber shapes (in 2D), allometry of chambers and three dimensional simulation space. The resulted "theoretical shells" closely mimics morphology and variability of recent and fossil foraminifera. The model is presented as an applet at http://www.icsr.agh.edu.pl/foraminifera.
\end{abstract}

\section{Introduction}

"Computer simulations are devised with the intention of mimicking nature and consequently improving the understanding of natural system" [1]. One of the greatest surprises of early theoretical morphologic modelling was that very complex organic forms could be produced by relatively simple mathematical models [2]. This is also the case with foraminifera - single-celled organisms, which build diverse and often complex shells. These protozoans, like many other organisms, grow by a process of discrete, stepwise additions of elements or parts accreted to an already existing morphology.

Majority of foraminifera are built of chambers (see Fig.1), which are cavities containing the protoplasm surrounded by a firm mineral wall [3]. The simplest shells are composed of a single chamber with a single opening (termed as aperture). The most complex ones build their chambers divided into smaller chamberlets with complex wall structures (see e.g. 456]). We focus on simple multilocular (multichambered) shells with single apertures, where every chamber composes a single opening. Old apertures in multilocular shells, which are called foramina, enable communication between all chambers, created during the growth of the shell (see Fig.11).

Modelling of foraminifera started very early with the classical work of Berger [7], which followed the first publication on theoretical morphology of accretive coiled shells [8] and first mathematical models of plant development [9]. The emergence of forms in the growth process of foraminiferal shells is an essential 
problem in ontogenesis of these microorganisms [5]6]. Our goal is to extend and develop the theoretical model of foraminiferal shell morphogenesis presented by Topa and Tyszka 56. This model has introduced a new approach to modelling of foraminiferal shells based on the moving reference system [56]. All former models referred to the fixed reference frame, represented by an invariable (fixed) coordinate system. The moving reference model is based on simple principles of motion and stepwise growth [10]. Such models have been developed for simulation of ammonites [11, but currently have also been applied for other organisms, such as plants or marine sessile organisms (e.g. [12,13]).

The Topa \& Tyszka model [5]6] uses real morphologic characters and follows stepwise natural biological processes. First of all, it introduces apertures as a fundamental morphogenetic feature of all foraminiferal shells. The aperture coordinates are based on minimisation of the local communication path, i.e. minimisation of the distance between successive openings (apertures) of chambers (see Fig. 11, d). This idea is partly derived from earlier studies of Hottinger [14] and Brasier [15], who noticed that foraminifera tend to shorten the global distance between the first and last compartment of its shell [5]6].

The purpose of this study is to extend functionality of Tyszka \& Topa model [56] by introducing: (1) 3D-space, (2) unrestricted (non-circular) shapes of chambers, (3) chamber allometry, (4) numerical methods of minimisation.

"Theoretical shell morphology requires hands-on experience to be appreciated. However, very little computer software for this field is publicly available" [16]. We want to fulfil this demand, thus, the application is available for research and educational purposes in the World Wide Web [17.

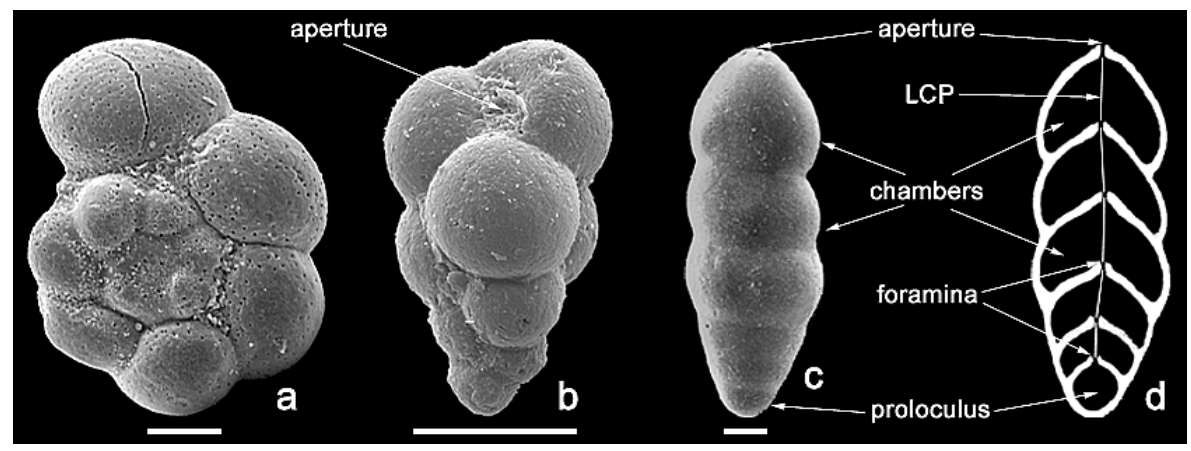

Fig. 1. Examples of foraminiferal morphotypes. (a) Hedbergella - spiral (low helicospiral) form; (b) Guembelitria - 3-serial (high helicospiral) form; (c, d) Pseudonodosaria uniserial form ([6] modified). LCP - Local Communication Path between two foramina of successive chambers. Scale bar $=50 \mu \mathrm{m}$. 


\section{The Model}

The iterative model we present in this paper is based on the concepts of moving reference system and minimisation of local communication path [56]. The reference system, in which the successive chambers are constructed, is not fixed to an arbitrary defined points or axes, but it changes its location along with the growth of the foraminifera shell. The distance between the apertures of the two successive chambers must be minimised. In opposition to the previous model [5], 6], the current model introduces chambers having any convex shape. Their contours are defined by a set of points (see Fig $2 \mathrm{a}$ and b). In consequence the "centre of the chamber" is chosen arbitrarily rather than in real geometric centre.

The application of different shapes of chambers makes difficult calculations of the aperture location. The application of analytical method, similarly to the previous model [56], is impossible. We have implemented a simple iterative method. The algorithm of the minimisation looks through the points forming a new chamber and calculates their distances to the aperture of the previous chamber. The point, which is placed nearest the previous aperture is chosen as an aperture of the new chamber. This method is not as precise as an analytical approach, but high precision is not a crucial requirement in this model.

a)



b)

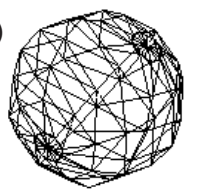

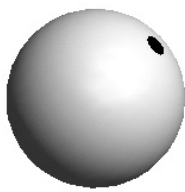

Fig. 2. Representation of chamber shape in a) 2D space, b) 3D space (black dot marks the aperture).

The condition is that a new aperture cannot be enclosed within any previous chamber, thus the algorithm checks whether the point lies inside the contour by counting the number of crossings of the line running through this point with that contour. An odd number of crossings means that the point lies inside the contour.

During the growth of the foraminifera, shapes of successive chambers can change. These changes are made by scaling the chambers in $x, y$ (and $z$ in $3 \mathrm{D}$ case) directions by appropriate coefficients.

\subsection{The Algorithm}

The algorithm uses the following concepts:

- centre of the chamber $-O_{i}$. It is the origin of the reference system as well.

- aperture point $-U_{i}$. It describes the location of chamber aperture. 
- growth vector $-\boldsymbol{v}_{i}$. It is attached to the aperture $U_{i}$ and points the centre of new chamber $O_{i+1}$.

- reference growth axis. It is a base direction for the growth vector $\boldsymbol{v}_{i}$.

We define the following parameters of simulation:

- deviation angle $-\Delta \phi_{i}$. It is an angle between the reference growth axis and the growth vector $\boldsymbol{v}_{i} ; \Delta \phi_{i} \in\left\langle-90^{\circ}, 90^{\circ}\right\rangle$.

- rotation angle $-\beta_{i}$. Angle by which the growth vector $\boldsymbol{v}_{i}$ is rotated around the reference growth axis; $\beta_{i} \in\left\langle-180^{\circ}, 180^{\circ}\right\rangle$, (only in the $3 \mathrm{D}$ case).

$-l_{i}^{\max }$ - maximum length of the growth vector $\boldsymbol{v}_{i}$, which cannot exceed this value, otherwise the new chamber would separate from the shell.

$-s_{i}$ - scaling rate of the growth vector $\boldsymbol{v}_{i}\left(0<s_{i}<0.9\right)$.

$-k_{i}^{x}$ - scaling rate of $x$ coordinate of points forming the chamber shape.

$-k_{i}^{y}$ - scaling rate of $y$ coordinate of points forming the chamber shape.

$-k_{i}^{z}$ - scaling rate of $z$ coordinate of points forming the chamber shape, (only in the $3 \mathrm{D}$ case).

Two-Dimensional Case. The simulation starts from the initial chamber. Its shape, size and orientation are arbitrarily defined. The location of the first aperture $U_{0}$ is calculated according to the local minimisation principle. The algorithm is searching for the point forming the chamber, which lies nearest the centre of the chamber. The initial reference growth axis is calculated as a line running through the points $O_{0}$ and $U_{0}$, i.e., the centre of the chamber and its aperture respectively. Figure 3 a shows the initial chamber.

a)

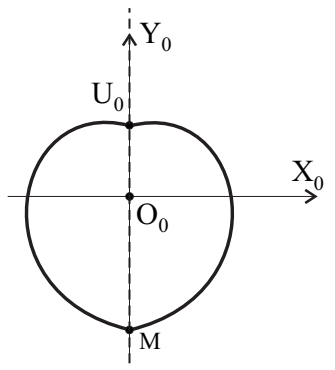

b)

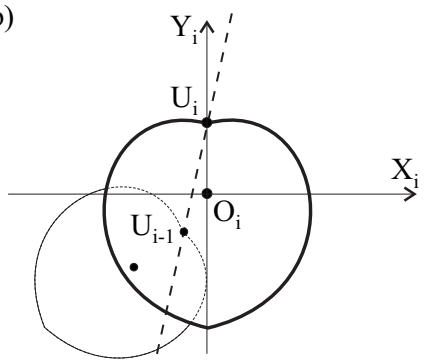

Fig. 3. The reference growth axis: a) for initial chamber, b)at further steps of simulation

At further steps of simulation, the reference growth axis is defined as a line running through the apertures of current and previous chambers i.e. $U_{i-1}$ and $U_{i}$ (see Fig. [3]).

The maximum length of the vector, $\boldsymbol{v}_{0}, l_{0}^{\max }$ is arbitrarily defined as equal to the length of the vector $\overline{O_{0} M}\left(l_{0}^{\max }=\left|\overline{O_{0} M}\right|\right) . M$ is the point in which the contour of initial chamber is crossing the negative part of $Y_{0}$ axis (see Fig. 3 a). 
For each step of the simulation, the procedure of calculating a new chamber consists of two phases:

1. Calculating the location and orientation of a new chamber:

a) the growth vector $\boldsymbol{v}_{i}$ is calculated first. It is attached to the aperture of the previous chamber $U_{i-1}$ and its direction is calculated by deviating it from the reference growth axis by angle $\Delta \phi_{i}$. The sense of the growth vector is consistent with the sense of the vector $\overline{U_{i-2} U_{i-1}}\left(\overline{O_{0} U_{0}}\right.$ for $i=1$ ) and its length is calculated as: $\left|\boldsymbol{v}_{i}\right|=s_{i} l_{i}^{\max }$, where $l_{i}^{\max }=k_{i}^{y} l_{i-1}^{\max }$. The growth vector $\boldsymbol{v}_{i}$ attached to a previous aperture $U_{i-1}$ points to a centre of new chamber $O_{i}$ (see Fig. $4 \mathrm{a}$ ).

b) $O_{i}$ point is also the origin of a new reference system. Positive $Y$ axis is defined by the direction of the growth vector $\boldsymbol{v}_{i}$ (see Fig. 4 $4 \mathrm{~b}$ ).

a)



b)

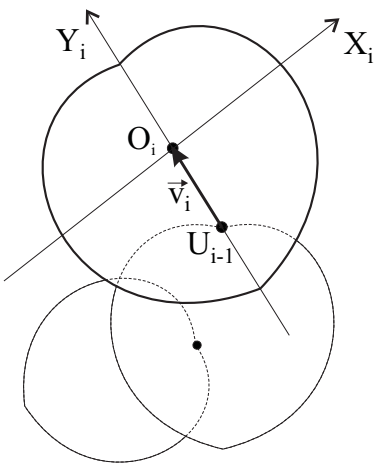

d)

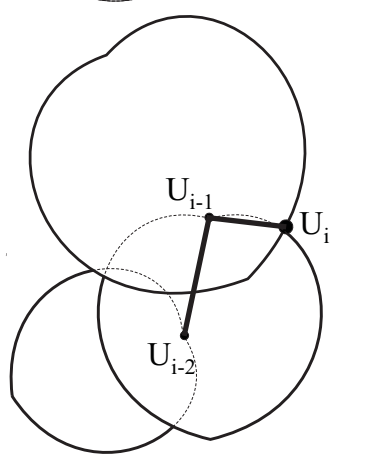

Fig. 4. Creation of the new chamber in $2 \mathrm{D}$ model: a) the growth vector, b) the new chamber, c) the reference growth axis, d) local communication path

2. Calculating the shape of a new chamber and finding its aperture:

a) the shape of the new chamber is calculated by scaling the geometry of the previous chamber in $x$ and $y$ direction by $k_{i}^{x}$ and $k_{i}^{y}$ coefficients, respectively. 
b) the location of a new aperture is calculated accordingly to the local minimisation paradigm. The distance between the apertures of that new chamber and the previous chamber must be the shortest. New aperture cannot be placed within any previous chamber.

The reference growth axis for the next iteration is calculated as a line running through the points $U_{i-1}$ and $U_{i}$ (see Fig. 4k).

The apertures of the successive chambers can be connected, creating the local communication path (see Fig. $4 \mathrm{~d}$ ).

Three-Dimensional Case. The algorithm in 3D case is similar to the 2D one. Again, the simulation starts from the initial chamber with arbitrarily defined shape, size and orientation. The location of the first aperture $U_{0}$ is calculated accordingly to the local minimisation principle. The initial reference growth axis is calculated as a line running through the points: $O_{0}$ and $U_{0}$ (see Fig. $5 \mathrm{a}$ ).

At further steps of simulation, the reference axis is calculated as a line running through the the last and previous aperture $U_{i}$ and $U_{i-1}$ (see Fig. 5b).

The maximum length of the vector $\boldsymbol{v}_{0}, l_{0}^{\max }$, is equal to the length of the vector $\overline{O_{0} M}$ (see Fig. 5 a).

For each step of simulation, the procedure of calculating a new chamber consist of the two phases:

1. Calculating the location and orientation of a new chamber.

a) the growth vector $\boldsymbol{v}_{i}$ is calculated first. It is handled at the aperture of the previous chamber $U_{i-1}$ and its direction is calculated by deviating it from the reference growth axis by angle $\Delta \phi_{i}$ and next rotating it around the reference axis by angle $\beta_{i}$. If $\beta_{i}=0^{\circ}$, the growth vector is included in the plain defined by points $U_{i-1}, U_{i-2}$ and $U_{i-3}$, i.e. three previous apertures (for $i=1$, in the plain defined by $O_{0}, U_{0}$ and perpendicular to plain $\left.O_{0} X_{0} Y_{0}\right)$. The sense of the growth vector is consistent with the sense of the vector $\overline{U_{i-2} U_{i-1}}\left(\overline{O_{0} U_{0}}\right.$ for $\left.i=1\right)$. The length of the growth vector is calculated as: $\left|\boldsymbol{v}_{i}\right|=s_{i} l_{i}^{\max }$, where $l_{i}^{\max }=k_{i}^{y} l_{i-1}^{\max }$. The growth vector $\boldsymbol{v}_{i}$ handled in $U_{i-1}$ aims a centre of new chamber $O_{i}$ (see Fig. 5 r).

b) this point $O_{i}$ becomes the origin of a new reference system. Positive $Y_{i}$ axis is defined by the direction of the growth vector $\boldsymbol{v}_{i}$.

2. Calculating the shape of a new chamber and finding its aperture.

a) the shape of a new chamber is calculated by scaling the geometry of the previous chamber in $x, y$ and $z$ coordinates by $k_{i}^{x}, k_{i}^{y}$ and $k_{i}^{z}$ coefficients, respectively.

b) the aperture of that new chamber must be as close to the aperture of the previous chamber as possible. It cannot be enclosed within any previous chamber (see Fig. 5d).

The reference growth axis for the next iteration is calculated as a line running through the points $U_{i-1}$ and $U_{i}$ (see Fig. $5 \mathrm{~d}$ ). 

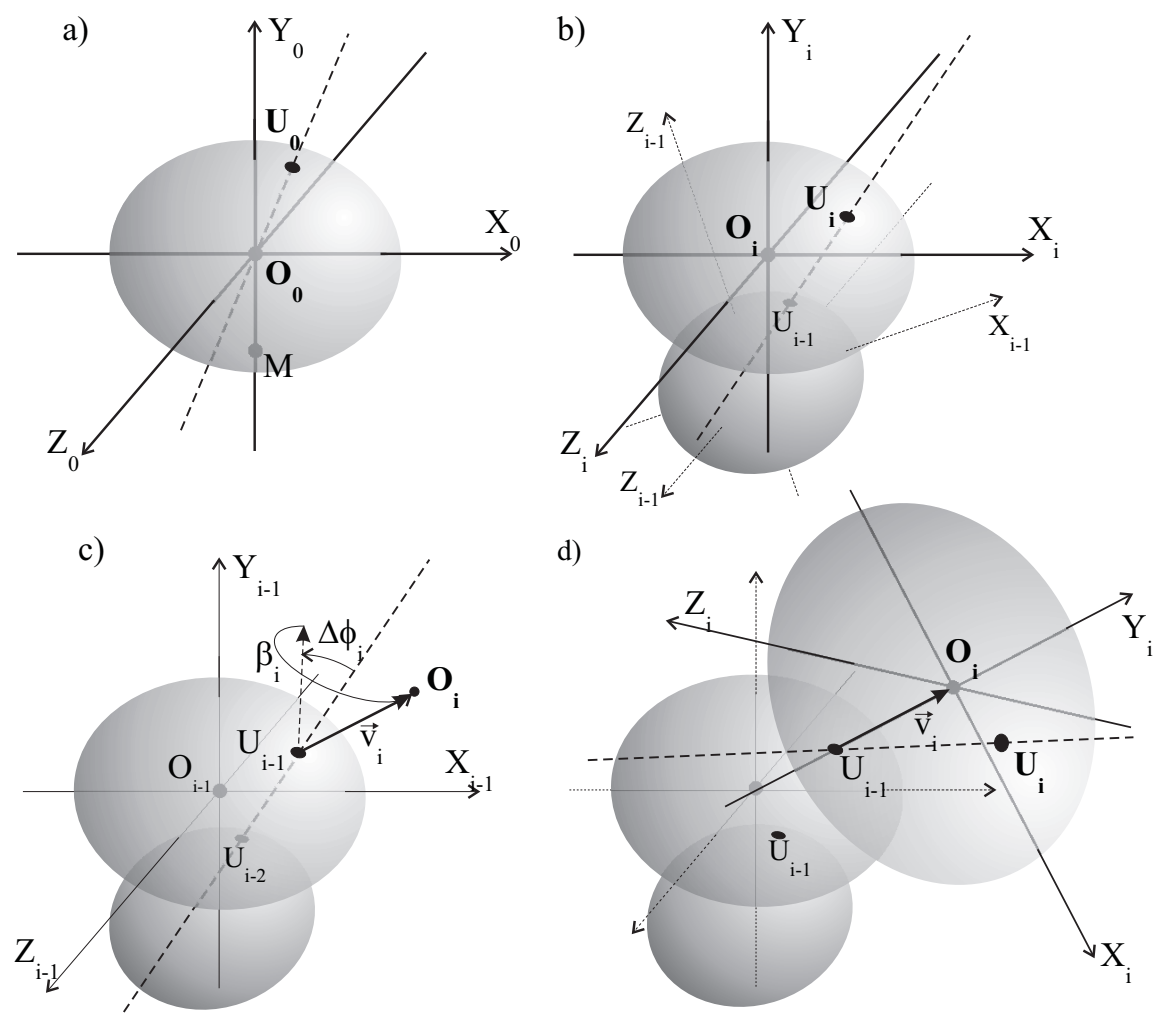

Fig. 5. 3D model: a) the reference growth axis for the initial chamber, b) at further steps of the simulation, c) the growth vector, d) the new chamber and its aperture.

\subsection{Implementation}

The model has been implemented as an Java applet with the Java3D library for visualisation purpose. It can be run from the WWW page [17] (see Fig. 66).

The applet allows users to manipulate most of parameters of the simulation $\left(k_{i}^{x, y, z}, s_{i}\right)$. In $2 \mathrm{D}$ case, the shape of the chamber can be chosen from the set of predefined contours. In $3 \mathrm{D}$ case, the only available shape of chamber is ellipsoidal.

\section{Discussion and Conclusions}

Our recent model follows the moving reference approach in theoretical modelling of foraminiferal shells. It extends functionality of the former model by application of new parameters leading to improvement of simulation results (see Figs. (7). The presented model introduces new elements, that were absent in the Topa \& Tyszka model. Some of them (3D model, allometric scaling of chambers) have been postulated in [5]6]. Due to introduction of chambers with various shapes, the numerical algorithm of minimisation had to be implemented instead of using 


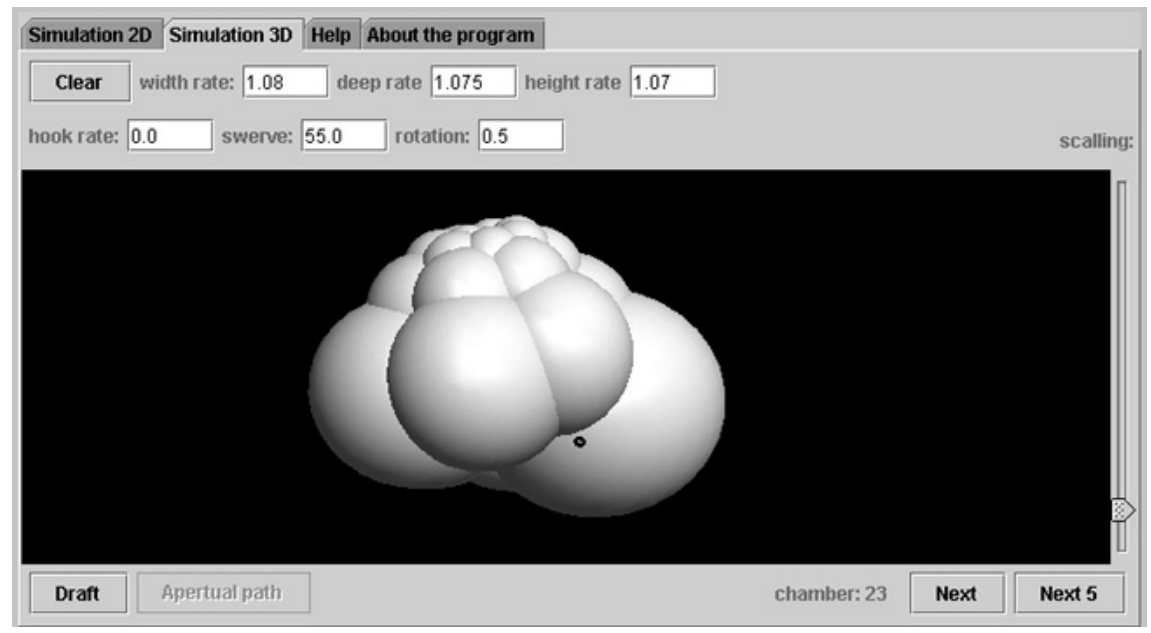

Fig. 6. Snapshot of the Java applet

analytical methods. In opposition to the Topa \& Tyszka model, the presented model does not use stochastic parameters, which should be applied in future (see Table 1 for comparison).

Table 1. Comparison of the presented model with Topa \& Tyszka [5]6] model

\begin{tabular}{|l|l|l|}
\hline & Topa \& Tyszka model & This model \\
\hline \hline $\begin{array}{l}\text { minimisation } \\
\text { method }\end{array}$ & analytical approach & numerical method \\
\hline dimensions & 2D only & 2D and 3D \\
\hline chambers & circular only & any shape \\
\hline $\begin{array}{l}\text { scaling of } \\
\text { chambers }\end{array}$ & isometric & allometric \\
\hline $\begin{array}{l}\text { parameters of } \\
\text { simulation }\end{array}$ & $\frac{T F_{i}-\text { chamber translation ratio }}{G F_{i}-\text { chamber expansion ratio }}$ & $\frac{s_{i}-\text { scaling rate of the growth vector }}{k_{i}^{x, y, z}-\text { chamber scaling rates }}$ \\
\cline { 2 - 3 } & $\frac{\Delta \phi_{i}-\text { deviation of the growth vector }}{\beta_{i}-\text { rotation of the growth vector }}$ \\
\hline \multirow{2}{*}{$\begin{array}{l}\text { selection of } \\
\text { parameters }\end{array}$} & stochastic or non-stochastic & non-stochastic \\
\hline
\end{tabular}

This model is an important step towards realistic modelling of foraminiferal shells and understanding of their morphogenetic processes. It extends complexity of the morphospace, which gets closer to reality (see Fig. 7). This aspect of the model should be investigated in future. The first attempt to the morphospace of the Topa \& Tyszka model can be found in [6]. However, this model exploits larger number of parameters and definition of its morphospace is much more complicated. Despite of significant improvements the presented model is still a 


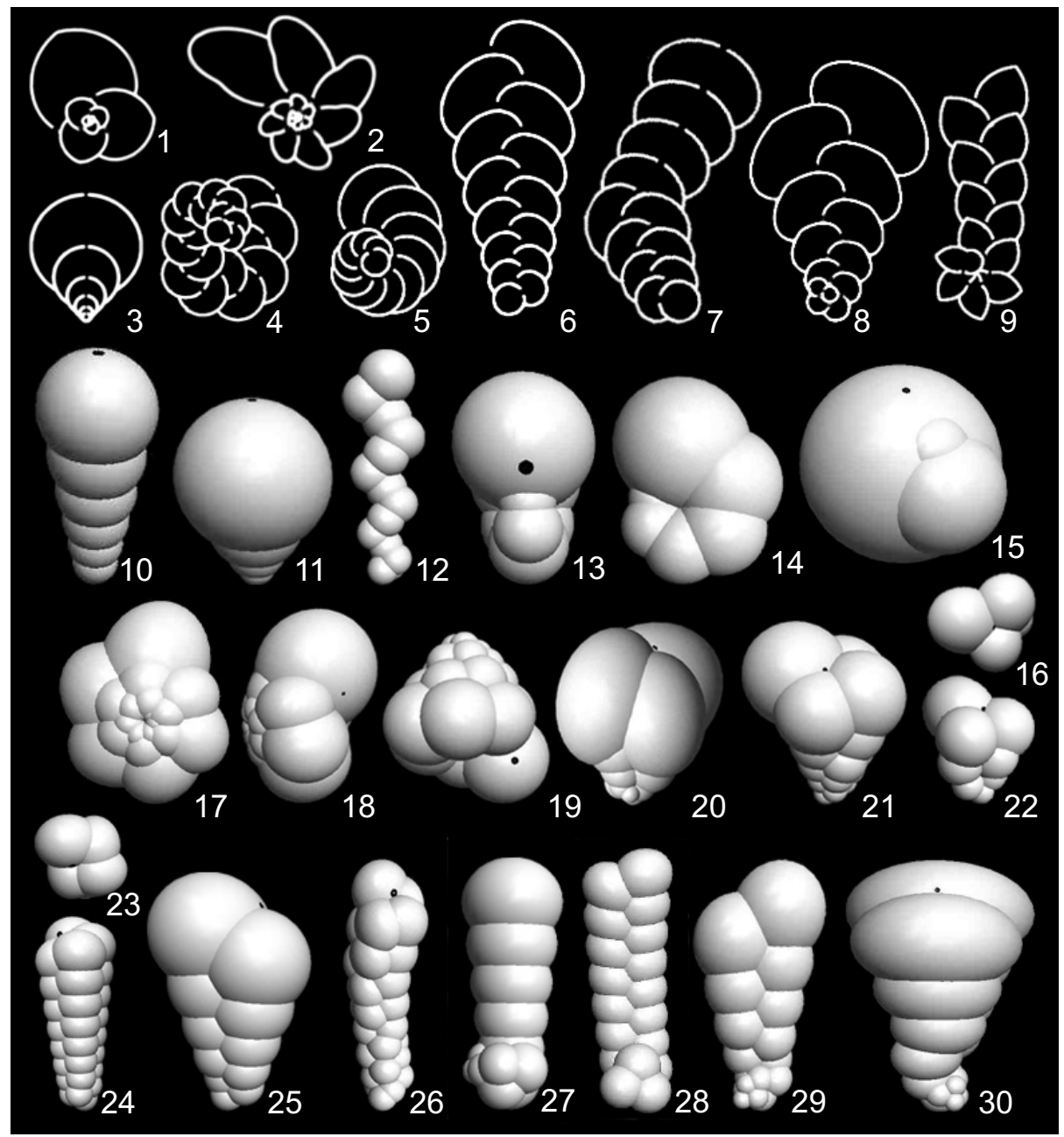

Fig. 7. Foraminiferal shells simulated by the applet in 2D (1-9) and 3D (10-30) with various arrangements, shapes, and number of chambers. $(1,2,5)$ spiral shells; (3) uniserial; (4) spiral with alternating chambers; (6) biserial; (7) biserial becoming uniserial; $(8,9)$ spiral becoming biserial; $(10,11)$ uniserial; (12) alternating uniserial; (13-15) planispiral forms; (17-24) various helicospiral forms; (25) biserial; (26) highly helicospiral switching from 5 to 4 rows; (27) streptospiral to uniserial; $(28,29,30)$ streptospiral (chaotic) to biserial. Not to scale. Black spots represents apertures.

serious simplification. In the next step, we plan to remove all elements, which are arbitrarily defined, e.g. calculating the maximum length of the growth vector. It would be also necessary to eliminate the parts of chambers which are enclosed in preceding chambers. This has not been solved so far, because it must be based on a different and more natural approach. 
The applet will be expanded by adding an extra base of predefined shapes of chambers prepared on the models of chambers that are met in the nature. The visualisation of the virtual foraminifera shells, mainly for the 3D model, should be also improved to make them look more realistic (e.g. by using suitable textures).

Acknowledgement. This research is partially sponsored by the Polish Committee for Scientific Research (KBN grant nr 3 PO4D 04824 awarded to JT and $\mathrm{PT})$.

\section{References}

[1] Swan, A.R.H., 1990. Computer simulation of invertebrate morphology. In: Bruton, D.L. \& Harper, D.A.T. (eds.), Microcomputers in Palaeontology. Contributions of the University of Oslo Palaeontological Museum no. 370: 32-45. Oslo.

[2] McGhee Jr., G.R., Theor. Morphol. The Concept and its Application. Perspectives in Paleobiology and Earth History, Columbia Univ. Press, New York, 1999.

[3] Lipps J.H., Fossil Prokaryotes and Protists, Blackwell, Boston, 1993.

[4] Hottinger L., Functional morphology of benthic foraminiferal shells, envelopes of cells beyond measure, Micropaleontology, 46:supplement no. 1, pp. 57-86, 2000.

[5] Topa P., Tyszka J., Local Minimisation Paradigm in Numerical Modelling of Foraminiferal Shells, in P.M.A Sloot et al. (Eds.) Proc. of the ICCS'2002, LNCS 2329, vol. 1, pp. 97-106, Amsterdam 2002.

[6] Topa P., Tyszka J., A new approach to modelling of morphogenesis of foraminiferal shells, Future Generation Computer Systems, Elsevier Science, in press.

[7] Berger W.H., Planktonic foraminifera: basic morphology and ecologic implications., Journal of Paleont., 6(43):1369-1383, 1969.

[8] Raup D.M., Michelson A, Theoretical morphology of the coiled shell, Science, 147:1294-1295, 1965.

[9] Lindenmayer A., Mathematical models for cellular interactions in development, I \& II. J. Theor. Biol., 18:280-315, 1968.

[10] Ackerly S.C, Kinematics of accretionary shell growth, with examples from brachiopods and molluscus, Paleobiology, 15:147-164, 1989.

[11] Okamoto T., Analysis of heteromorph ammonoids by differential geometry, Palaeontology,31:35-52, 1988.

[12] Prusinkiewicz P., Lindenmayer A., The Algorithmic Beauty of Plants, SpringerVerlag, New York, 1990.

[13] Kaandorp J.A., A formal description of radiate accretive growth, J. Theor. Biol., 166:149-161, 1994.

[14] Hottinger L., Comparative Anatomy of Elementary Shell Structures in Selected Larger Foraminifera, In: Hedley, R.H. and Adams, C.G. (eds.) Foraminifera, 3:203-266, 1978.

[15] Brasier, Foraminiferid architectural history; a review using the MinLOC and PI methods, J. of Micropaleontology, 1:95-105, 1982.

[16] Savazzi, E., 1995. Theoretical shell morphology as a tool in constructional morphology. Neues Jahrbuch für Geologie und Paläontologie. Abhandlungen, 195:229240.

[17] http://www.icsr.agh.edu.pl/foraminifera 